\title{
Prediction of the Spectrophotometric Response of a Carded Fiber Composed by Different Kinds of Coloured Raw Materials: An Artificial Neural Network-Based Approach
}

Rocco Furferi and Lapo Governi

\begin{abstract}
One of the most critical phases of the production of carded fiber is the comparison between the colour of the finished product and the colour desired by a customer or provided in a catalog. In most cases, the required proportions of coloured fibers for obtaining a desired final colour of a fiber are evaluated by trial and error techniques. This approach, which often lacks a computer-aided tool to assist colourists, allows the assessment of a recipe to reproduce a target colour. The companies producing carded fibers often perform a colorimetric control of their product. This is obtained by evaluating the colorimetric distance between the final product, obtained by mixing some coloured raw fibers in order to obtain a homogeneous colour, and the desired one. When this colorimetric distance exceeds a value stated by the customer, the companies have to modify the recipe in order to reduce the gap between the colour of the final product and the desired one.
\end{abstract}

The present work describes a novel approach for a reliable prediction of the spectrophotometric response of a carded fiber based on the spectrophotometric response of the raw materials composing the fiber itself. This is obtained for any variation of an original recipe. The developed system is, first, based on a theoretical approach used for the prediction of reflectance factors obtained by mixing the same kind of differently coloured raw material. A more general approach, based on Artificial Neural Networks (ANNs), is then described. This second approach performs a reliable prediction of the reflectance factors of a carded fiber composed by several kind of differently coloured raw materials.

The two approaches were used to predict the reflectance factors of 500 different blends. Colour differences between the predicted reflectance factors and the real spectrophotometric response of the carded fibers are measured and expressed in CIELAB and CMC(2:1) distances under several standard illuminants. Referring to the test performed in the present work, the mean predicted colour difference in terms of CIELAB is less than 0.24 . The maximum distance in terms of $\mathrm{CMC}(2: 1)$ is less than 0.25 . Experimental validation demonstrates that the proposed approach is suitable for a reliable, fast and practical prediction of the recipes for carded fibers.

For these reasons, the developed system is actually running into the Laboratory of an important textile company located in Prato (Italy): the New Mill S.p.A. 